\title{
Modified Fractional Variational Iteration Method for Solving the Generalized Time-Space Fractional Schrödinger Equation
}

\author{
Baojian Hong ${ }^{1,2}$ and Dianchen $\mathrm{Lu}^{1}$ \\ ${ }^{1}$ Faculty of Science, Jiangsu University, Zhenjiang, Jiangsu 212013, China \\ ${ }^{2}$ Department of Basic Causes, Nanjing Institute of Technology, Nanjing 211167, China \\ Correspondence should be addressed to Baojian Hong; hbj@njit.edu.cn
}

Received 8 May 2014; Revised 24 July 2014; Accepted 27 July 2014; Published 4 September 2014

Academic Editor: Mustafa Inc

Copyright (C) 2014 B. Hong and D. Lu. This is an open access article distributed under the Creative Commons Attribution License, which permits unrestricted use, distribution, and reproduction in any medium, provided the original work is properly cited.

\begin{abstract}
Based on He's variational iteration method idea, we modified the fractional variational iteration method and applied it to construct some approximate solutions of the generalized time-space fractional Schrödinger equation (GFNLS). The fractional derivatives are described in the sense of Caputo. With the help of symbolic computation, some approximate solutions and their iterative structure of the GFNLS are investigated. Furthermore, the approximate iterative series and numerical results show that the modified fractional variational iteration method is powerful, reliable, and effective when compared with some classic traditional methods such as homotopy analysis method, homotopy perturbation method, adomian decomposition method, and variational iteration method in searching for approximate solutions of the Schrödinger equations.
\end{abstract}

\section{Introduction}

In the past decades, due to the numerous applications of fractional differential equations (FDEs) in the areas of nonlinear science [1], many important phenomena can be described successfully using the FDEs models such as materials and processes [2], engineering and physics [3], dielectric polarization [4], and quantitative finance [5]. Searching for solutions of these FDEs plays an important and significant role in all aspects of this subject. But because of the complexity of nonlinear terms and fractional derivative, it is very difficult for us to obtain the exact analytic solutions of most FDEs, so approximate and numerical methods must be considered. A great deal of efforts have been proposed for these problems, like the homotopy analysis method (HAM) [6], the homotopy perturbation method (HPM) [7], the adomian decomposition method (ADM) [8], the generalized differential transform method [9], and so forth [10].

The variational iteration method (VIM) established in 1999 by He in [11] is thoroughly used by many researchers to construct the approximate solutions of a wide variety of scientific and engineering models [12, 13]. After some modifications, the fractional variational iteration method (FVIM) was applied to fractional differential equations by He and many authors [14-19]. The motivation of this paper is to construct some analytical approximate solutions for the GFNLS powerfully. Firstly, we give some modifications for the FVIM and extend the application of the FVIM. Secondly, we use the modified fractional variational iteration method (MFVIM) to the GFNLS and compare the efficiency of MFVIM with some other traditional perturbation methods. The results show that MFVIM gives rapid and standard convergence to the exact solution if such a solution exists.

We give some basic definitions and properties of the fractional calculus theory which are used further in this paper; we define the following fractional integral and derivatives $[20,21]$.

Definition 1. A real function $f(x)$ is said to be in the space $C_{\mu}$, where $\mu \in R, x>0$, if there exists a real number $p(>\mu)$ such that $f(x)=x^{p} f_{1}(x)$, where $f_{1}(x) \in C[0, \infty)$ and it is said to be in the space $C_{\mu}^{m}$ if and only if $f^{(m)} \in C_{\mu}, m \in N$. 
Definition 2. The Riemann-Liouville fractional integral operator of order $\alpha>0$ for a function $f(x) \in C_{\mu}, \mu \geq-1$, is defined as follows:

$$
\begin{aligned}
& J_{x}^{\alpha} f(x)= \frac{1}{\Gamma(\alpha)} \int_{0}^{x}(x-\xi)^{\alpha-1} f(\xi) d \xi \\
&= \frac{1}{\Gamma(\alpha+1)} \int_{0}^{x} f(\xi)(d \xi)^{\alpha}, \quad \alpha>0, x>0, \\
& J_{x}^{0} f(x)=f(x) .
\end{aligned}
$$

$$
J^{\alpha} x^{\gamma}=\frac{\Gamma(\gamma+1)}{\Gamma(\alpha+\gamma+1)} x^{\alpha+\gamma} \text {. }
$$

Definition 3. For $\alpha>0, x>0$, and $f(x) \in C_{-1}^{n}$, the Caputo fractional derivative operator of order $\alpha$ on the whole space is defined as follows:

$$
\begin{aligned}
D^{\alpha} f(x) & =J^{n-\alpha} D^{n} f(x) \\
& = \begin{cases}\frac{1}{\Gamma(n-\alpha)} \int_{0}^{x}(x-\xi)^{n-\alpha-1} f^{(n)}(\xi) d \xi, \\
\frac{d^{(n)} f(x)}{d x^{n}}, & \alpha=n .\end{cases}
\end{aligned}
$$

Also one has the following properties:

$$
\begin{gathered}
D^{\alpha} C=0, \quad(C \text { is a constant }), \\
D^{\alpha} x^{\gamma}= \begin{cases}\frac{\Gamma(\gamma+1)}{\Gamma(\gamma-\alpha+1)} x^{\gamma-\alpha}, & \gamma>\alpha-1, \\
0, & \gamma \leq \alpha-1,\end{cases} \\
J^{\alpha} D^{\alpha} f(x)=f(x)-\sum_{k=0}^{n-1} f^{(k)}\left(0^{+}\right) \frac{x^{k}}{k !}, \\
n-1<\alpha<n, \\
D^{\alpha} J^{\alpha} f(x)=f(x) .
\end{gathered}
$$

\section{Analysis of the MFVIM and the FGNLS}

Consider the following generalized time and space fractional nonlinear Schrödinger equation with variable coefficients $[22,23]$ :

$$
\begin{gathered}
i \frac{\partial^{\alpha} u}{\partial t^{\alpha}}+a \frac{\partial^{2 \beta} u}{\partial x^{2 \beta}}+v(x) u+\gamma|u|^{2} u=0, \\
t>0, \quad 0<\alpha, \quad \beta \leq 1, \\
u(x, 0)=f(x),
\end{gathered}
$$

where $u=u(x, t), \partial^{\alpha} u / \partial t^{\alpha}=D_{t}^{\alpha} u, \partial^{2 \beta} u / \partial x^{2 \beta}=D_{x}^{\beta}\left(D_{x}^{\beta} u\right)$, $v(x)$ is the trapping potential, and $a, \gamma$ are the slowly increasing dispersion coefficient and nonlinear coefficient, respectively. If we select $\alpha=\beta=1, v(x)=0$, this equation turns to the famous nonlinear Schrödinger equations in optical fiber [24-26].

According to the FVIM [14-19], we can build a correction functional for (5) as follows:

$$
\begin{aligned}
& u_{n+1}= u_{n}+\frac{1}{\Gamma(1+\alpha)} \\
& \times \int_{0}^{t} \lambda(\tau, x)\left[i \frac{\partial^{\alpha} u_{n}}{\partial t^{\alpha}}+a \frac{\partial^{2 \beta} \widetilde{u}_{n}}{\partial x^{2 \beta}}\right. \\
&\left.+v(x) \widetilde{u}_{n}+\gamma \widetilde{u}_{n}\left|\tilde{u}_{n}\right|^{2}\right](d \tau)^{\alpha},
\end{aligned}
$$

with the initial condition $u_{0}=u(x, 0)=f(x)$, where $\lambda(t, x)$ is a general Lagrange's multiplier which can be identified optimally with the variational theory. The function $\widetilde{u}_{n}$ is a restricted variation which means $\delta \widetilde{u}_{n}=0$. Therefore, we first determine the Lagrange multiplier $\lambda(t, x)$ that will be identified optimally via integration by parts [27]. The successive approximations $u_{n+1}, n \geq 0$, of the solution $u(x, t)$ will be readily obtained through $\lambda(t, x)$ and any selective function $u_{0}$. The initial values are usually used for choosing the zeroth approximation $u_{0}$. With $\lambda(t, x)$ determined, then several approximations $u_{k}, k=1,2, \ldots$ follow immediately. Consequently, the exact solution may be procured by using $u=\lim _{n \rightarrow \infty} u_{n}$. The convergence of FVIM has been proved in [28]. In this paper, notice that (5) is a complex differential equation with complex modulus term $|u|^{2}$, as we all know, a complex function $u(\xi)$ can be written as $c(\xi) e^{i \theta(\xi)}$, where $c(\xi)$ and $\theta(\xi)$ are real functions, noticed that $|u(\xi)|^{2}=|c(\xi)|^{2}$, we can give some modification for the iteration formulation (6), assume that $\lim _{n \rightarrow \infty}\left|\tilde{u}_{n}\right|^{2}=|u|^{2}=\left|u_{0}\right|^{2}$, we get the MFVIM for (5). This modification should enhance rapidly the efficiency of our iteration.

In what follows, in order to illustrate the strength of this method, we will apply the MFVIM to some models about (5).

\section{Approximate Solutions for the FGNLS}

Example 4. We first consider the time-fractional NLS equation [29]:

$$
\begin{aligned}
i \frac{\partial^{\alpha} u}{\partial t^{\alpha}}+a \frac{\partial^{2} u}{\partial x^{2}}+\gamma u|u|^{2} & =0, \quad t>0,0<\alpha \leq 1, \\
u(x, 0) & =f(x) .
\end{aligned}
$$

The correction functional for (7) reads

$$
\begin{aligned}
u_{n+1}= & u_{n}+\frac{1}{\Gamma(1+\alpha)} \\
& \times \int_{0}^{t} \lambda(\tau, x)\left[i \frac{\partial^{\alpha} u_{n}}{\partial t^{\alpha}}+a \frac{\partial^{2} \widetilde{u}_{n}}{\partial x^{2}}+\gamma \widetilde{u}_{n}\left|\widetilde{u}_{n}\right|^{2}\right](d \tau)^{\alpha} .
\end{aligned}
$$


Making the above correction functional stationary,

$$
\begin{aligned}
\delta u_{n+1}= & \delta u_{n}+\lambda(t, x) i \delta u_{n} \\
& -\frac{1}{\Gamma(1+\alpha)} \int_{0}^{t}\left[i D_{\tau}^{(\alpha)} \lambda(\tau, x) \delta u_{n}\right](d \tau)^{\alpha} .
\end{aligned}
$$

After getting the coefficients of $\delta u_{n}$ to zero we can determine the Lagrange multiplier

$$
\lambda=i
$$

We produce the iteration formulation as follows:

$$
u_{n+1}=u_{n}+\frac{i}{\Gamma(1+\alpha)} \int_{0}^{t}\left[i \frac{\partial^{\alpha} u_{n}}{\partial \tau^{\alpha}}+a \frac{\partial^{2} u_{n}}{\partial x^{2}}+\gamma u_{n}\left|u_{0}\right|^{2}\right](d \tau)^{\alpha}
$$

As stated before, we can select $u_{0}=u(x, 0)=$ Asec $h x$; using the iteration (11) and the mathematica software, we obtain the following successive approximations:

$$
\begin{aligned}
& u_{0}=A \sec h x \text {, } \\
& u_{1}=A \operatorname{sech} x+a A i\left(\operatorname{sech} x-2 \operatorname{sech}^{3} x\right) \frac{1}{\Gamma(1+\alpha)} t^{\alpha} \\
& +A^{3} i \gamma \sec ^{3} x \frac{1}{\Gamma(1+\alpha)} t^{\alpha} \\
& =A \operatorname{sech} x\left[1+\frac{a i t^{\alpha}}{\Gamma(1+\alpha)}\right] \\
& +\left(-2 a+A^{2} \gamma\right) A i \sec h^{3} x \frac{1}{\Gamma(1+\alpha)} t^{\alpha} \\
& =A \operatorname{sech} x\left[1+\frac{a i t^{\alpha}}{\Gamma(1+\alpha)}\right], \quad\left(A^{2}=\frac{2 a}{\gamma}\right), \\
& u_{2}=A \operatorname{sech} x+a A i\left(\operatorname{sech} x-2 \operatorname{sech}^{3} x\right) \\
& \times\left[\frac{1}{\Gamma(1+\alpha)} t^{\alpha}+a i \frac{1}{\Gamma(1+2 \alpha)} t^{2 \alpha}\right] \\
& +\gamma A^{3} i \operatorname{sech} h^{3} x\left[\frac{1}{\Gamma(1+\alpha)} t^{\alpha}+a i \frac{1}{\Gamma(1+2 \alpha)} t^{2 \alpha}\right] \\
& =A \operatorname{sech} x\left[1+\frac{a i t^{\alpha}}{\Gamma(1+\alpha)}+\frac{1}{\Gamma(1+2 \alpha)}\left(\text { ait }^{\alpha}\right)^{2}\right] \text {, } \\
& u_{3}=A \operatorname{sech} x\left[1+\frac{a i t^{\alpha}}{\Gamma(1+\alpha)}+\frac{\left(a i t^{\alpha}\right)^{2}}{\Gamma(1+2 \alpha)}+\frac{\left(a i t^{\alpha}\right)^{3}}{\Gamma(1+3 \alpha)}\right] \text {, } \\
& u_{n}=A \operatorname{sech} x \sum_{k=0}^{n} \frac{1}{\Gamma(1+k \alpha)}\left(a i t^{\alpha}\right)^{k}
\end{aligned}
$$

The exact solution of (7) is

$$
\begin{aligned}
& u=A \operatorname{sech} x \lim _{n \rightarrow \infty} \sum_{k=0}^{n} \frac{1}{\Gamma(1+k \alpha)}\left(a i t^{\alpha}\right)^{k} \\
& = \pm \sqrt{\frac{2 a}{\gamma}} \operatorname{sech} x E_{\alpha}\left(a i t^{\alpha}\right),
\end{aligned}
$$

where $E_{\alpha}\left(a i t^{\alpha}\right)$ is the Mittag-Leffler function. If we let $\alpha=1$ in (13), the exact solution of the regular NLS equation (7) can be obtained as follows:

$$
\left.u\right|_{\alpha=1}= \pm \sqrt{\frac{2 a}{\gamma}} \operatorname{sech} x e^{i a t}
$$

Example 5. We now consider the time-space fractional NLS equation $[30,31]$ :

$$
\begin{gathered}
i \frac{\partial^{\alpha} u}{\partial t^{\alpha}}+a \frac{\partial^{2 \beta} u}{\partial x^{2 \beta}}+2 a u|u|^{2}=0, \\
t>0, \quad 0<\alpha, \quad \beta \leq 1, \\
u(x, 0)=e^{i x} .
\end{gathered}
$$

With the similar process, we get the iteration formulation as follows:

$$
\begin{aligned}
u_{n+1}= & u_{n}+\frac{i}{\Gamma(1+\alpha)} \\
& \times \int_{0}^{t}\left[i \frac{\partial^{\alpha} u_{n}}{\partial \tau^{\alpha}}+a \frac{\partial^{2 \beta} u_{n}}{\partial x^{2 \beta}}+2 a u_{n}\left|u_{0}\right|^{2}\right](d \tau)^{\alpha} .
\end{aligned}
$$

Using the iteration (16) and the mathematica software, we obtain the following successive approximations:

$$
\begin{aligned}
& u_{0}=e^{i x} \\
& u_{1}=e^{i x}\left[1+\frac{\left(2+e^{i \pi \beta}\right) i a t^{\alpha}}{\Gamma(1+\alpha)}\right] \\
&=e^{i x}\left(1+\frac{c_{1} i a t^{\alpha}}{\Gamma(1+\alpha)}\right), \quad c_{1}=2+e^{i \pi \beta}, \\
& u_{2}=e^{i x}\left\{1+\frac{\left(2+e^{i \pi \beta}\right) i a t^{\alpha}}{\Gamma(1+\alpha)}\right. \\
&+\left[\left(2+e^{i \pi \beta}\right) e^{i \pi \beta}\right. \\
&\left.\left.+2\left(2+e^{i \pi \beta}\right)\right] \frac{i^{2} a^{2} t^{2 \alpha}}{\Gamma(1+2 \alpha)}\right\}
\end{aligned}
$$




$$
\begin{gathered}
=e^{i x}\left[1+\frac{c_{1} i a t^{\alpha}}{\Gamma(1+\alpha)}+\frac{c_{1}^{2} i^{2} a^{2} t^{2 \alpha}}{\Gamma(1+2 \alpha)}\right], \\
u_{3}=e^{i x}\left\{1+\frac{\left(2+e^{i \pi \beta}\right) i a t^{\alpha}}{\Gamma(1+\alpha)}\right. \\
+\left(2 c_{1}+c_{1} e^{i \pi \beta}\right) \frac{i^{2} a^{2} t^{2 \alpha}}{\Gamma(1+2 \alpha)} \\
\left.+\left(2 c_{1}^{2}+c_{1}^{2} e^{i \pi \beta}\right) \frac{i^{3} a^{3} t^{3 \alpha}}{\Gamma(1+3 \alpha)}\right\} \\
=e^{i x}\left[1+\frac{c_{1} i a t^{\alpha}}{\Gamma(1+\alpha)}+\frac{c_{1}^{2} i^{2} a^{2} t^{2 \alpha}}{\Gamma(1+2 \alpha)}+\frac{c_{1}^{3} i^{3} a^{3} t^{3 \alpha}}{\Gamma(1+3 \alpha)}\right], \\
\vdots \quad \\
u_{n}=e^{i x} \sum_{k=0}^{n} \frac{1}{\Gamma(1+k \alpha)}\left[i\left(2+e^{i \pi \beta}\right) a t^{\alpha}\right]^{k} .
\end{gathered}
$$

The exact solution of (15) is

$$
\begin{aligned}
u & =e^{i x} \lim _{n \rightarrow \infty} \sum_{k=0}^{n} \frac{1}{\Gamma(1+k \alpha)}\left[i\left(2+e^{i \pi \beta}\right) a t^{\alpha}\right]^{k} \\
& =e^{i x} E_{\alpha}\left[i\left(2+e^{i \pi \beta}\right) a t^{\alpha}\right] .
\end{aligned}
$$

If we let $\alpha=1$ and let $\beta=1$ in (18), the exact solution of the regular NLS equation (15) can be obtained as follows:

$$
\left.u\right|_{(\alpha=1, \beta=1)}=e^{i(x+a t)} .
$$

Remark 6. The solution (18) is more standard than the result (3.18) in [30]. If one selects $a=1$ or $a=1 / 2$, the solution (19) is the same as the result (49) in [29], the result (3.21) in [30], and the result (29) in [31], but one can find that this iteration is much more standard and powerful than the HAM, the ADM, and the VIM mentioned in [29-31].

Example 7. Consider the following time-space fractional NLS equation [23]:

$$
\begin{aligned}
& i \frac{\partial^{\alpha} u}{\partial t^{\alpha}}+\frac{1}{2} \frac{\partial^{2 \beta} u}{\partial x^{2 \beta}}-u \cos ^{2} x-|u|^{2} u=0, \\
& t>0, \quad 0<\alpha \leq 1, \quad u(x, 0)=\sin x .
\end{aligned}
$$

With the similar process, we get the iteration formulation as follows:

$$
\begin{aligned}
u_{n+1}= & u_{n}+\frac{i}{\Gamma(1+\alpha)} \\
& \times \int_{0}^{t}\left[i \frac{\partial^{\alpha} u_{n}}{\partial \tau^{\alpha}}+\frac{1}{2} \frac{\partial^{2 \beta} u_{n}}{\partial x^{2 \beta}}-u_{n} \cos ^{2} x-u_{n}\left|u_{0}\right|^{2}\right](d \tau)^{\alpha} .
\end{aligned}
$$

If we select $u_{0}=u(x, 0)=\sin x$, using the iteration (21) and the mathematica software, we obtain the following successive approximations:

$$
\begin{aligned}
u_{0}= & \sin x \\
u_{1}= & \sin x+\left[\frac{1}{2} \sin (x+\pi \beta)-\sin x\right] \frac{i t^{\alpha}}{\Gamma(1+\alpha)} \\
u_{2}= & \sin x+\left[\frac{1}{2} \sin (x+\pi \beta)-\sin x\right] \frac{i t^{\alpha}}{\Gamma(1+\alpha)} \\
& +\left[\frac{1}{4} \sin (x+2 \pi \beta)-\sin (x+\pi \beta)+\sin x\right] \\
& \times \frac{i^{2} t^{2 \alpha}}{\Gamma(1+2 \alpha)}, \\
u_{3}= & u_{2}+\left[\frac{1}{8} \sin (x+3 \pi \beta)-\frac{3}{4} \sin (x+2 \pi \beta)\right. \\
& \left.\quad+\frac{3}{2} \sin (x+\pi \beta)-\sin x\right] \frac{i^{3} t^{3 \alpha}}{\Gamma(1+3 \alpha)}
\end{aligned}
$$

$$
u_{n}=\sum_{k=0}^{n} c_{k}(x) \frac{\left(i t^{\alpha}\right)^{k}}{\Gamma(1+k \alpha)},
$$

where $c_{k}(x)=c_{k, 0} \sin x+c_{k, 1} \sin (x+\pi \beta)+c_{k, 2} \sin (x+$ $2 \pi \beta)+\cdots+c_{k, k} \sin (x+k \pi \beta), c_{k, 0}=(-1)^{k}, c_{k, 1}=$ $(1 / 2) c_{k-1,1}-c_{k-1,0}, \ldots, c_{k, k-1}=(1 / 2) c_{k-1, k-2}-c_{k-1, k-1}$, and $c_{k, k}=(1 / 2) c_{k-1, k-1}, k \geq 2, c_{0,0}=1 ; c_{1,0}=-1, c_{1,1}=(1 / 2)$; $c_{2,0}=1, c_{2,1}=-1, c_{2,2}=(1 / 4) ; \ldots$

The exact solution of $(20)$ is

$$
\begin{aligned}
u & =\lim _{n \rightarrow \infty} \sum_{k=0}^{n} c_{k}(x) \frac{\left(i t^{\alpha}\right)^{k}}{\Gamma(1+k \alpha)} \\
& =\sin \left[\frac{x^{\beta}}{\Gamma(1+\beta)}\right] \operatorname{Exp}\left[-\frac{3}{2} \frac{i t^{\alpha}}{\Gamma(1+\alpha)}\right] .
\end{aligned}
$$

If we let $\alpha=1$ and let $\beta=1$ in (23), the exact solution of the regular NLS equation (20) can be obtained as follows.

$$
\left.u\right|_{(\alpha=1, \beta=1)}=\sin x e^{-(3 / 2) i t} .
$$

If we select $u_{0}=u(x, 0)=\cos x$, with the same process, we can also obtain the following exact solution of (20):

$$
u=i \cos \left[\frac{x^{\beta}}{\Gamma(1+\beta)}\right] \operatorname{Exp}\left[-\frac{1}{2} \frac{i t^{\alpha}}{\Gamma(1+\alpha)}\right] .
$$

Remark 8. If one selects $\beta=1$, the solution (23) is more standard than the result (5.10) in [23]. The solutions (23) and (25) are new exact solutions for (20) to our knowledge.

Comparisons between the real part of some numerical results and the exact solution (23) are summarized in Tables 1 and 2 , and the simulations for $u_{4}, u_{\mathrm{abs}}$, and $u$ are plotted in Figures 1 and 2, which shows that the MFVIM produced a rapidly convergent series. 
TABLE 1: Comparison between the real part of $u_{4}$ and $u$ when $\alpha=\beta=1$.

\begin{tabular}{lcccc}
\hline$x$ & $t$ & Approximate solution $u_{\text {4appr }}$ & Exact solution & Absolute error \\
\hline 1 & 0.4 & 0.6945501509 & 0.6944959727 & 0.0000541782 \\
5 & 0.4 & -0.7914960963 & -0.7914343559 & 0.0000617404 \\
1 & 0.3 & 0.7577097797 & 0.7577001100 & $9.66968 \times 10^{-6}$ \\
15 & 0.3 & 0.5855572741 & 0.5855498014 & $7.47272 \times 10^{-6}$ \\
12 & 0.2 & -0.5126082301 & -0.5126076876 & $6.16597 \times 10^{-6}$ \\
3 & 0.2 & 0.13481723570 & 0.13481709305 & $1.42655 \times 10^{-7}$ \\
2 & 0.1 & 0.8990870113 & 0.8990869969 & $1.43796 \times 10^{-8}$ \\
0.2 & 0.1 & 0.1964384915 & 0.1964384884 & $3.14175 \times 10^{-9}$ \\
\hline
\end{tabular}

TABLE 2: Comparison between the real part of $u_{4}$ and $u$ when $\alpha=0.7, \beta=0.9$.

\begin{tabular}{lcccc}
\hline$x$ & $t$ & Approximate solution $u_{\text {4appr }}$ & Exact solution & Absolute error \\
\hline 1 & 0.4 & 0.5356787528 & 0.5565185584 & 0.02083980558 \\
2 & 0.4 & 0.5092408025 & 0.6018550029 & 0.0926142003 \\
1 & 0.3 & 0.6273816439 & 0.6535277868 & 0.02614614289 \\
2 & 0.3 & 0.6242882934 & 0.7067670289 & 0.08247873543 \\
1 & 0.2 & 0.7153235950 & 0.7417557951 & 0.02643220012 \\
0.2 & 0.2 & 0.1978265247 & 0.2080382267 & 0.01021170194 \\
0.2 & 0.1 & 0.1989280524 & 0.2288404399 & 0.02991238755 \\
12 & 0.1 & 0.8741657132 & 0.8902825407 & 0.01611682753 \\
\hline
\end{tabular}

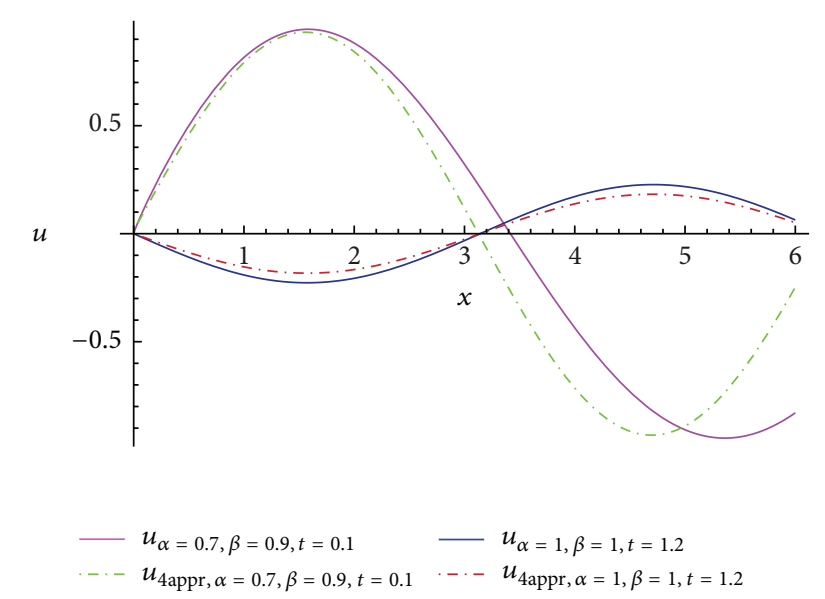

FIgURE 1: Comparison between the real part of $u_{4}$ and the exact solution $u$.

\section{Summary}

In this paper, the MFVIM is used for finding approximate and exact solutions of the GFNLS equation with Caputo derivative. The obtained results indicate that the MFVIM is effective, convenient, and powerful method for solving nonlinear fractional complex differential equations when comparing it with some other traditional asymptotic decomposition methods such as HAM, VIM, and ADM. We believe that these methods should play an important role for finding exact and approximate solutions in the mathematical physics.

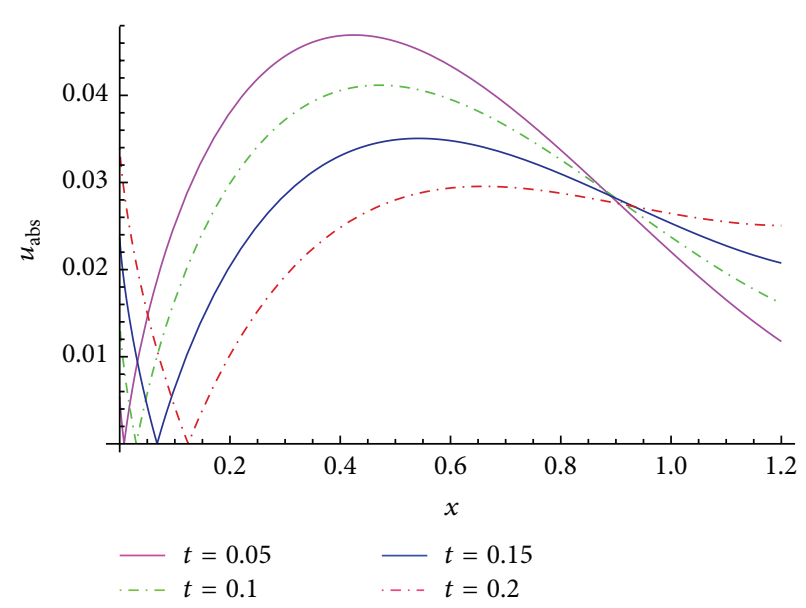

FIGURE 2: Plots of the absolute error $u_{\mathrm{abs}}$ when $\alpha=0.7$ and $\beta=0.9$.

\section{Conflict of Interests}

The authors declare that there is no conflict of interests regarding the publication of this paper.

\section{Acknowledgments}

This paper is supported by the National Nature Science Foundation of China (no. 61070231); the Outstanding Personal Program in Six Fields of Jiangsu (2009188); and the General Program of Innovation Foundation of NanJing Institute of Technology (Grant no. CKJB201218). 


\section{References}

[1] M. Dalir and M. Bashour, "Applications of fractional calculus," Applied Mathematical Sciences, vol. 4, pp. 1021-1032, 2010.

[2] I. Podlubny, Fractional Differential Equations, Academic Press, New York, NY, USA, 1999.

[3] K. S. Miller and B. Ross, An Introduction to the Fractional Calculus and Fractional Differential Equations, John Wiley \& Sons, New York, NY, USA, 1993.

[4] H. H. Sun, A. A. Abdelwahab, and B. Onaral, "Linear approximation of transfer function with a pole of fractional order," IEEE Transactions on Automatic Control, vol. 29, no. 5, pp. 441-444, 1984.

[5] N. Laskin, "Fractional market dynamics," Physica A, vol. 287, no. 3-4, pp. 482-492, 2000.

[6] S. Panda, A. Bhowmik, R. Das, R. Repaka, and S. C. Martha, "Application of homotopy analysis method and inverse solution of a rectangular wet fin," Energy Conversion and Management, vol. 80, pp. 305-318, 2014.

[7] K. A. Gepreel, “The homotopy perturbation method applied to the nonlinear fractional Kolmogorov-Petrovskii-Piskunov equations," Applied Mathematics Letters, vol. 24, no. 8, pp. 14281434, 2011.

[8] L. N. Song and W. G. Wang, "A new improved Adomian decomposition method and its application to fractional differential equations," Applied Mathematical Modelling, vol. 37, no. 3, pp. 1590-1598, 2013.

[9] Z. Odibat, S. Momani, and V. S. Erturk, "Generalized differential transform method: application to differential equations of fractional order," Applied Mathematics and Computation, vol. 197, no. 2, pp. 467-477, 2008.

[10] M. Inc and A. Akgül, "Numerical solution of seventh-order boundary value problems by a novel method," Abstract and Applied Analysis, vol. 2014, Article ID 745287, 9 pages, 2014.

[11] J. H. He, "Variational iteration method-a kind of non-linear analytical technique: some examples," International Journal of Non-Linear Mechanics, vol. 34, no. 4, pp. 699-708, 1999.

[12] M. A. Noor and S. T. Mohyud-Din, "Variational iteration method for solving higher-order nonlinear boundary value problems using He's polynomials," International Journal of Nonlinear Sciences and Numerical Simulation, vol. 9, pp. 141-156, 2008.

[13] S. Momani and S. Abuasad, "Application of He's variational iteration method to Helmholtz equation," Chaos, Solitons \& Fractals, vol. 27, no. 5, pp. 1119-1123, 2006.

[14] J. H. He, "Some applications of nonlinear fractional differential equations and their approximations," Bulletin of Science, Technology \& Society, vol. 15, no. 2, pp. 86-90, 1999.

[15] J. He, "A short remark on fractional variational iteration method," Physics Letters A, vol. 375, no. 38, pp. 3362-3364, 2011.

[16] M. Merdan, "A numeric-analytic method for time-fractional Swift-Hohenberg (S-H) equation with modified RiemannLiouville derivative," Applied Mathematical Modelling. Simulation and Computation for Engineering and Environmental Systems, vol. 37, no. 6, pp. 4224-4231, 2013.

[17] G. C. Wu, "A fractional variational iteration method for solving fractional nonlinear differential equations," Computers \& Mathematics with Applications, vol. 61, no. 8, pp. 2186-2190, 2011.

[18] G. C. Wu and D. Baleanu, "Discrete fractional logistic map and its chaos," Nonlinear Dynamics, vol. 75, no. 1-2, pp. 283-287, 2014.
[19] G. C. Wu and D. Baleanu, "Variational iteration method for the Burgers' flow with fractional derivatives-new Lagrange multipliers," Applied Mathematical Modelling, vol. 37, no. 9, pp. 6183-6190, 2013.

[20] M. Inc, "The approximate and exact solutions of the spaceand time-fractional Burgers equations with initial conditions by variational iteration method," Journal of Mathematical Analysis and Applications, vol. 345, no. 1, pp. 476-484, 2008.

[21] M. G. Sakar, F. Erdogan, and A. Yıldırım, "Variational iteration method for the time-fractional Fornberg-Whitham equation," Computers \& Mathematics with Applications, vol. 63, no. 9, pp. 1382-1388, 2012.

[22] M. Ganjiani, "Solution of nonlinear fractional differential equations using homotopy analysis method," Applied Mathematical Modelling. Simulation and Computation for Engineering and Environmental Systems, vol. 34, no. 6, pp. 1634-1641, 2010.

[23] S. Z. Rida, H. M. El-Sherbiny, and A. A. M. Arafa, "On the solution of the fractional nonlinear Schrödinger equation," Physics Letters A, vol. 372, no. 5, pp. 553-558, 2008.

[24] R. Hao, L. Li, Z. Li, W. Xue, and G. Zhou, "A new approach to exact soliton solutions and soliton interaction for the nonlinear Schrödinger equation with variable coefficients," Optics Communications, vol. 236, no. 1-3, pp. 79-86, 2004.

[25] Y. Chen and B. Li, "An extended subequation rational expansion method with symbolic computation and solutions of the nonlinear Schrödinger equation model," Nonlinear Analysis: Hybrid Systems, vol. 2, no. 2, pp. 242-255, 2008.

[26] B. Li and Y. Chen, "On exact solutions of the nonlinear Schrödinger equations in optical fiber," Chaos, Solitons and Fractals, vol. 21, no. 1, pp. 241-247, 2004.

[27] R. Almeida and D. F. M. Torres, "Calculus of variations with fractional derivatives and fractional integrals," Applied Mathematics Letters, vol. 22, no. 12, pp. 1816-1820, 2009.

[28] S. Yang, A. Xiao, and H. Su, "Convergence of the variational iteration method for solving multi-order fractional differential equations," Computers \& Mathematics with Applications, vol. 60, no. 10, pp. 2871-2879, 2010.

[29] M. Ganjiani, "Solution of nonlinear fractional differential equations using homotopy analysis method," Applied Mathematical Modelling, vol. 34, no. 6, pp. 1634-1641, 2010.

[30] M. A. E. Herzallah and K. A. Gepreel, "Approximate solution to the time-space fractional cubic nonlinear Schrodinger equation," Applied Mathematical Modelling, vol. 36, no. 11, pp. 56785685, 2012.

[31] A. Wazwaz, "A study on linear and nonlinear Schrodinger equations by the variational iteration method," Chaos, Solitons and Fractals, vol. 37, no. 4, pp. 1136-1142, 2008. 


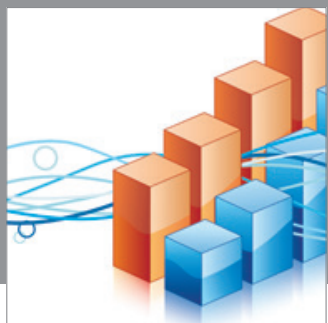

Advances in

Operations Research

mansans

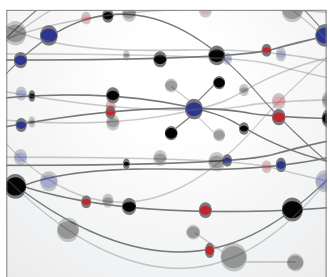

The Scientific World Journal
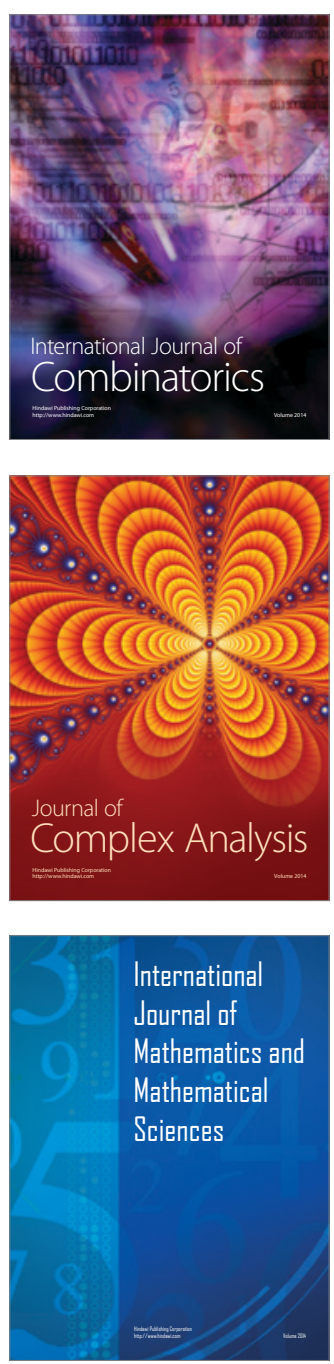
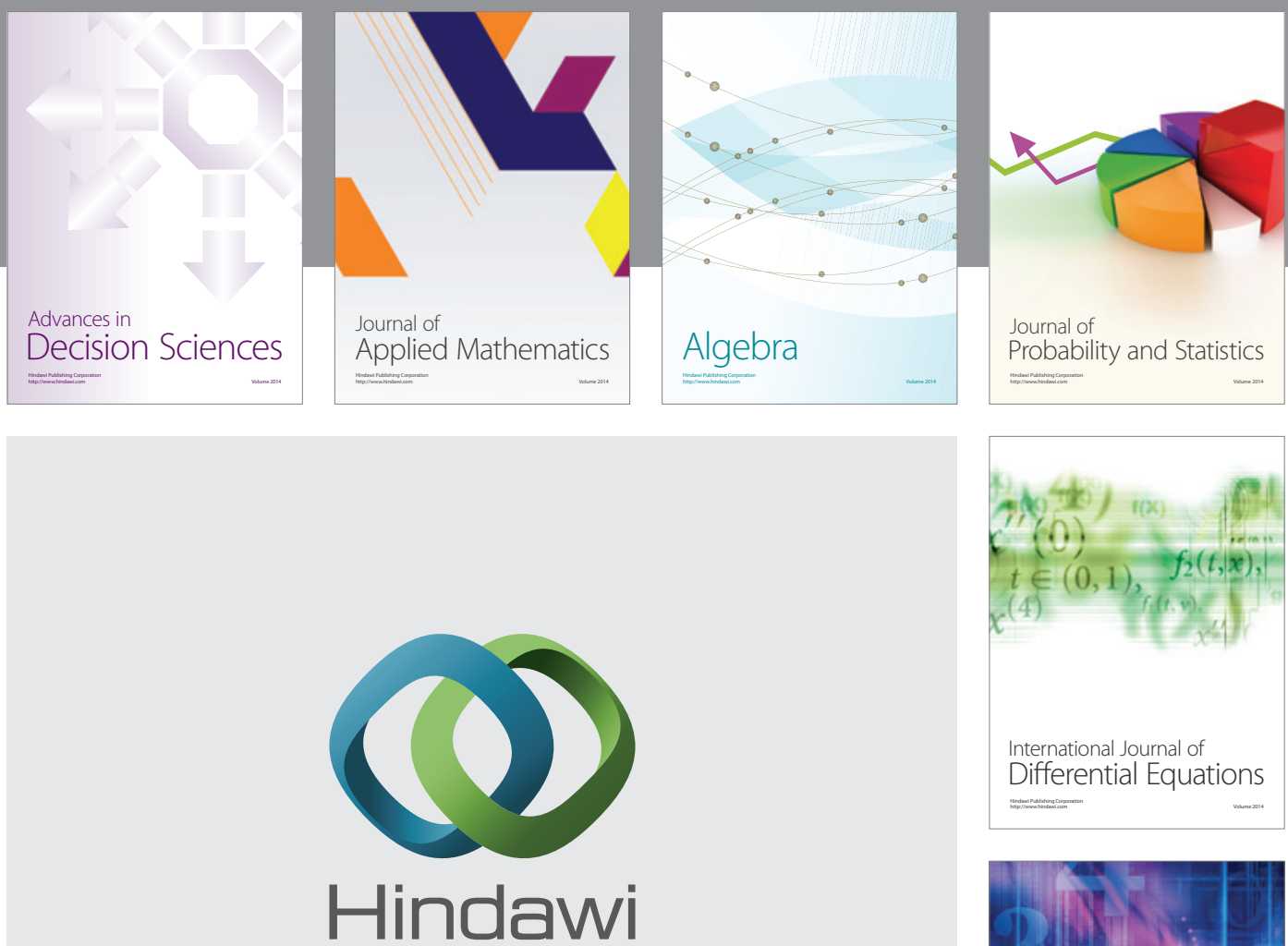

Submit your manuscripts at http://www.hindawi.com
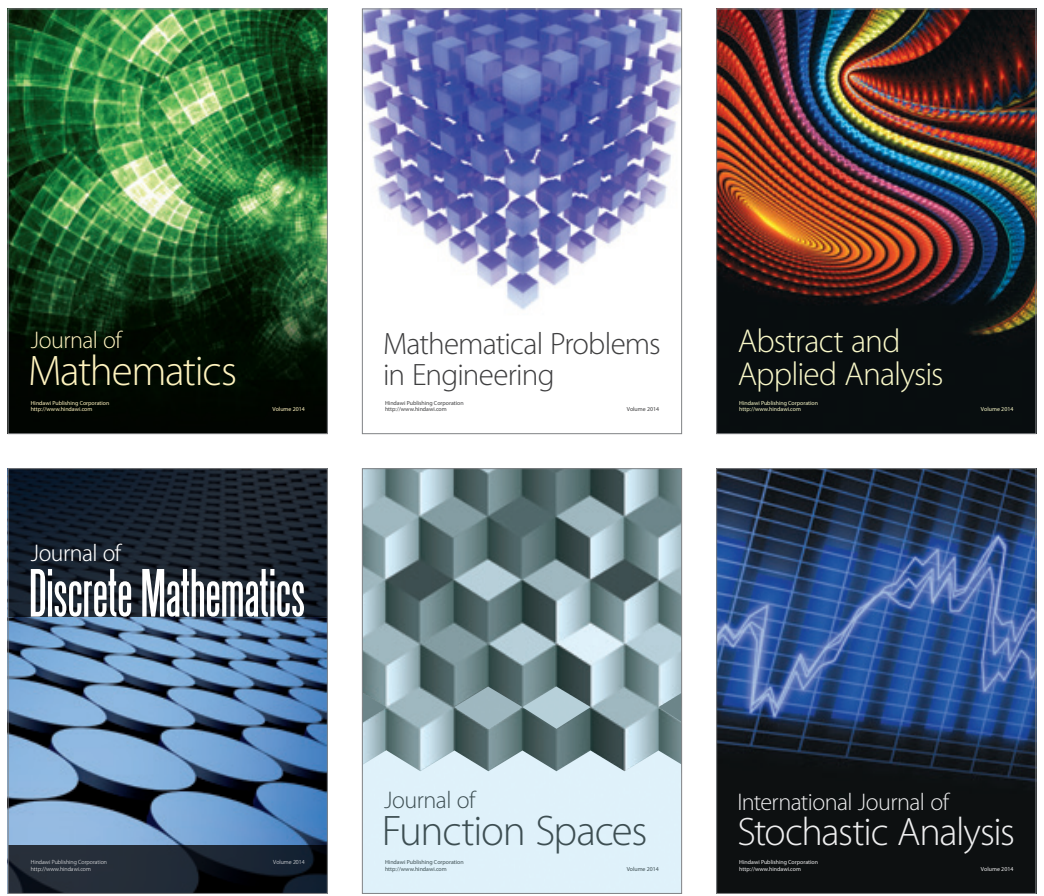

Journal of

Function Spaces

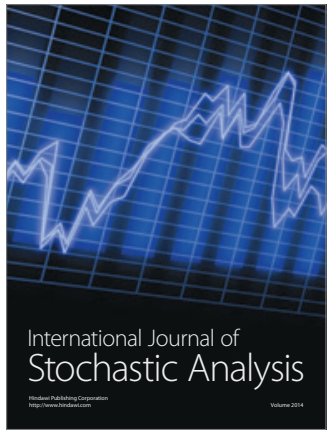

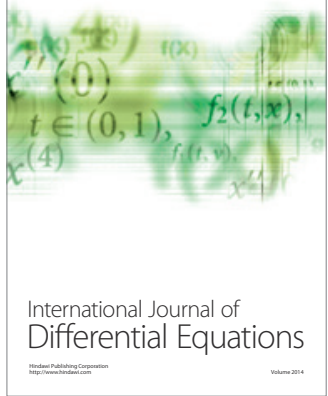
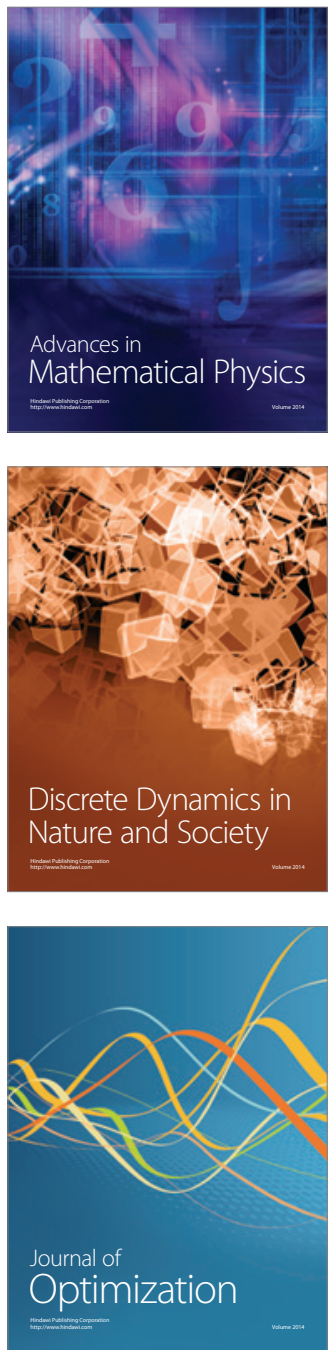